\title{
Investigation and Analysis on the Characteristics of Five Personalities of the Depressed Patients
}

\author{
Zhen Li \\ Graduate School \\ Jiangxi University of Traditional Chinese Medicine \\ Nanchang, Jiangxi, China \\ 493819785@qq.com \\ Tian-lin Chen* \\ Psychological Counseling Center \\ Jiangxi University of Traditional Chinese Medicine \\ Nanchang, Jiangxi, China \\ 88110440@qq.com
}

\author{
Man-li Jiang \\ School of Humanities \\ Jiangxi University of Traditional Chinese Medicine \\ Nanchang, Jiangxi, China
}

\author{
Lan Luo \\ School of Humanities \\ Jiangxi University of Traditional Chinese Medicine \\ Nanchang, Jiangxi, China
}

\begin{abstract}
For the investigation of the characteristics of the depressed patients, this paper tests 52 depressed patients by means of the Big Five Personality Test Scale. And the results obtained show the scores of the depressed patients in the personality dimension of Tai-yang, Shao-yang, Yin-Yang Balance are significantly lower than that of the national normal; There is no significant difference in the Shao-yin personality dimension between the depressed patients and the national normal; The scores of the depressed patients in Tai-yin personality dimension are significantly higher than that of the national normal. Moreover, the scores of the male depressed patients in the personality dimension of Tai-yang, Shao-yang, Yin-Yang Balance, Shao-yin are significantly lower than that of the female patients while the scores in Tai-yin personality dimension are significantly higher than that of the female patients. And it is noticeable that the personality dimension of Tai-yin accounts for a dominant position among all the personality dimensions for depressed patients, and the male depressed patients are more serious than the female patients when it comes to the pathological abnormality
\end{abstract}

Keywords-Depressed Patients; The Five State Personality; Personality Characteristics; Investigation

\section{INTRODUCTION}

With the change of modern society, people's stress in work, study and life are increasing. Depression has become the most common mental disorder in the world. Depression is a major type of mood disorder. It is characterized by a significant and persistent mood depression which is not commensurate with its position, often exhibiting diminished energy, loss of interest, low self evaluation or guilt, even grief, and repeated suicidal thinking and behavior, and more serious patients may have mental symptoms, Such as stiffness, hallucinations, delusions, etc. [1].

At present, both at home and abroad, the study of scholars agree that the personality characteristics of the depressed patients are mainly manifested in emotional instability, introversion and neuroticism. As for the psychological

Project supported by the subject of Science and Technology of Jiangxi

Provincial Education Department (NO. JXYJG-2016-0820)

Corresponding author: Tian-lin CHEN characteristics of the depressed patients, it is a consistent view that the depressed patients tend to be introverted, emotionally unstable and neurotic. There is a certain correlation between the onset of depression and its psychological characteristics. Their psychological characteristics and the way of attribution play an important role in the pathogenesis process [2]. In the current clinical work, personality assessment is based on internationally recognized personality assessment scales such as MMPI and EPQ. But these test scale based on western psychological theories is inaccurate to describe and explain Chinese behavior and personality characteristics. Therefore, Chong-cheng XUE and other people set up The Five State Personality Test Scale based on Chinese cultural background and national conditions, which has become a classical Chinese personality scale tailored for Chinese people. This scale has been widely applied in medicine, education, management and so on. It has become one of the general psychological test methods in China and has been widely accepted by the famous psychologists and the experts of Neijing.

Personality has always been the difficulty and key of psychotherapy. In order to prevent depression effectively, the key is to provide targeted psychological guidance and intervention on the basis of understanding the psychological characteristics of the individual, so then to promote the personality maturity of the individual. Therefore, the investigation of the personality characteristics of the five state of depressed patients and the analysis of its personality characteristics from the perspective of Chinese traditional culture will help to provide a new perspective for the treatment of the depressed patients, and it also help to promote the localization of Chinese psychotherapy. 


\section{SUBJECTS AND METHODS}

\section{A. Research subjects}

The depressed patients in two hospitals in Nanchang were selected as subjects according to ICD-10 diagnostic criteria and without severe suicidal tendencies. A total of 60 questionnaires were distributed and 52 valid questionnaires were collected, with an effective rate of $86.7 \%$. 52 effective subjects included 19 male patients and 33 female patients.

\section{B. Research methods}

\section{1) Five States Personality Test Scale}

The Five State Personality Test Scale compiled by Professor Chong-cheng XUE [3] was used as a research tool. The scale is based on the discussion of Yin-Yang and five states in Neijing of Chinese medicine, combining with the Chinese cultural characteristics and the Chinese norm. The five state personality scale divides people into five states: Taiyang, Shao-yang, Yin-Yang Balance, Shao-yin, and Tai-yin.

We adopted The Five State Personality Test Scale, which was compiled by Chong-cheng XUE and other professors of the Chinese academy of Chinese medical sciences. The five state personality test scale holds that five state people represent the intensity, flexibility, balance, persistence and progression of people's response to things from the perspective of characteristics theory. The personality characteristics of the five state people are as follows: the type of Tai-yang is characterized by fortitude, courage, boldness, courage, insistence on his own opinion, enterprising, unafraid of striking, subjective, ambitious, willful and irrelevant, irascible and irritable, impulsive, dared, arrogant, obstinately, and so on. The type of Shao-yang is characterized by best communication, good social interaction, optimistic, easygoing, witty, more action, careless, frivolous, like talking, laughing and recreational activities, and so on. The type of Yin-Yang Balance is characterized by calm, dignity and humility, indeed invisible, not affected by material and confused, selfless fearless, not complacent, live quiet and order, get carried away, regardless of gain and loss, follow the law of the development of things, etc. What's more, this type possesses a kind of character with high level of balance. The type of Shao-ying is characterized by caution, carefulness, coldness, vigilance, tolerance, persistence, jealousy, etc.The type of Tai-yin is characterized by modesty, misgivings, overcast, more thinking before action, unwilling to take the lead, unwilling to contact people, lonely, pessimistic, cowardly, keeping a certain distance from people, dislike excitement, disagreement, conservation, selfishness and so on.
There were six sub-scales and 103 test questions in the five personality test scale. There are 20 questions in the Taiyang scale, 22 questions in the Shao-yang scale, 10 questions in the Yin-Yang Balance scale, 21 questions in the Shao-yin scale, 22 questions in the Tai-yin scale and 8 questions in the disguised scale. For all the questions in the five personality test scale, if the answer is "yes", you will get 1 points for each question. The scores of each scale will be calculated after the test. When the score of disguised scale is less than 5 , it is invalid as a questionnaire.

\section{2) Data collection and processing}

The investigators were accepted the training of psychological measurement knowledge and unified instruction. Before conducting the questionnaire, we explained the purpose and significance of the survey, and got the trust and cooperation of the subjects. The method of anonymous evaluation is applied to enable the subjects to complete the questionnaire independently according to their actual situations and real feelings in the prescribed time. The patients who had missed or repeated assessments were reminded to reevaluate the results, so as not to affect the objectivity and accuracy of the results. In the process of questionnaire survey, the experimenters were required to answer doubts about the questionnaire content and the way of answering toward subjects. SPSS19.0 software was used for statistical analysis.

\section{RESULTS}

1) General Situation of Five State Personality Characteristics of the Depressed Patients

The results of the study showed that the average score of depression in the personality dimension of the Tai-yang was 11.28, which was significantly lower than the average score of 13.21 in the normal model control group (national norm) ( $\mathrm{t}=-$ 12.298, $\mathrm{P}<0.01)$. The average score in the Shao-yang dimension was 9.41 , which was significantly lower than the average score of 12.33 in the normal model group ( $\mathrm{t}=-15.842$, $\mathrm{P}<0.01)$. The average score in the Yin-Yang Balance was 4.75 , which was also significantly lower than the average score of 6.32 in the normal model control group $(\mathrm{t}=-16.314, \mathrm{P}<0.01)$. The average score in the personality dimension of the Shaoyin was 13.22, and there was no statistical difference between the average score of the normal model group $(\mathrm{t}=-0.487$, $\mathrm{P}<0.01)$. The average score in the personality dimension of the Tai-yin was 13.54 , significantly higher than the average score of 9.80 in the normal model control group $(\mathrm{t}=18.783, \mathrm{P}<0.01)$. See Table 1 specifically.

TABLE I THE FIVE STATE PERSONALITY OF DEPRESSION PATIENTS COMPARED WITH THE NATIONAL NORM

\begin{tabular}{|c|c|c|c|}
\hline & Depression Group & National Norm Group & $\mathrm{t}$ \\
\hline Taiy-ang & $11.28 \pm 3.59$ & $13.21 \pm 3.60$ & $-12.298^{* *}$ \\
\hline Shao-yang & $9.41 \pm 4.12$ & $12.33 \pm 4.21$ & $-15.842^{* *}$ \\
\hline Yin-Yang Balance & $4.75 \pm 2.41$ & $6.32 \pm 2.35$ & $-16.314^{* *}$ \\
\hline Shao-yin & $13.22 \pm 3.23$ & $13.62 \pm 3.77$ & -0.487 \\
\hline Tai-yin & $13.54 \pm 4.59$ & $9.80 \pm 4.99$ & $18.783^{* *}$ \\
\hline
\end{tabular}


2) Gender Comparison of Five Personality Characteristics of the Depressed Patients

Gender differences in five state personality characters in of depressed patients are as follows: the average score of male patients in Tai-yang personality dimension was 10.88, which was significantly lower than the score of 11.43 of the female patients $(\mathrm{t}=-2.513, \mathrm{P}<0.01)$. The average score of male patients in Shao-yang dimension was 9.02, which was significantly lower than the average score of 9.93 in female patients $(\mathrm{t}=-3.094, \mathrm{P}<0.01)$. The average score of male patients in the dimension of Yin-Yang Balance was 4.34, which was significantly lower than the average score of 4.85 in female patients $(\mathrm{t}=-3.121, \mathrm{P}<0.01)$. The average score of male patients in the personality dimension of Shao-yin was 13.05, which was significantly lower than the average score of 13.84 in female patients $(\mathrm{t}=-3.125, \mathrm{P}<0.01)$. The average score of male patients in Tai-yin personality dimension was 14.61, which was significantly higher than the average score of 12.93 in female patients $(\mathrm{t}=5.796, \mathrm{P}<0.01)$. See Table 2 specifically.

Table 2 Gender Comparison of Five Personality Characteristics of the Depressed Patients

\begin{tabular}{|c|c|c|c|}
\hline & male patients & female patients & t \\
\hline Taiy-ang & $10.88 \pm 3.21$ & $11.43 \pm 3.66$ & $-3.094^{* * *}$ \\
\hline Shao-yang & $9.02 \pm 3.91$ & $9.93 \pm 4.15$ & $-3.121^{* *}$ \\
\hline Yin-Yang Balance & $4.34 \pm 2.21$ & $4.85 \pm 2.38$ & $-3.125^{* *}$ \\
\hline Shao-yin & $13.05 \pm 3.22$ & $13.84 \pm 3.42$ & $5.796^{* *}$ \\
\hline
\end{tabular}

\section{DISCUSSION}

The study found that the score of the depressed patients in Tai-yang, Shao-yang, Yin-Yang Balance are significantly lower than that of the national norm group, but the score of the depressed patients was significantly higher than that of the national norm group in the personality dimension of Tai-yin. This is similar to the results of Meng-han LUI [4] et al. That is to say, in this study, the five state personality characteristics of the depressed patients are mainly Tai-yin people with less "Yang" and more "Yin".

This result is very similar to the major clinical features of the depressed patients. According to the main clinical characteristics of the patients with depression, the personality characteristics of the depressed patients are mainly similar to the negative characteristics such as "introverted silence, selfmade introverted, forbearance worry" and so on. There is a clear lack of positive characteristics such as "extrovert, bravery, agility, arrogance," and so on. This also illustrates the difference in coping style between depression patients and ordinary people due to personality. As a matter of fact, Neijing has been discussed about the relationship between physique and personality more than two thousand years ago. It is believed that because of the different $Q i$ content of Yin and Yang in the human body, the characteristics of human consciousness, temperament, character and behavior are different, and it is also emphasized that the difference between Yin and Yang of the body will also will also affect people's mental state, behavior and ability to deal with the society.

Chinese medicine holds that Yin and Yang are both opposites and unity. Yin and Yang are the basic foundation of the physical and mental health of human. The character of a normal person is both Yin and Yang. They can have multiple personality characteristics at the same time, but it is different in tendency. Depression is a Yin disease in traditional Chinese medicine. It is of great significance to stimulate Yang $Q i$ for its treatment. Research in recent years shows that Qi-gong exercise combined with dynamic and static exercises can improve depression and other negative emotional experiences. dynamic exercises make the body strong, and static exercises trengthens the consciousness. Exercise helps to generate Yang $Q i$, while quiet helps to maintain Yin Qi. Therefore, the treatment of depression, combined with the results of this study, can start from static and dynamic exercises to boost Yang $Q i$ and balance Yin and Yang in the body, So as to improve the depressive symptoms and personality characteristics of patients. Therefore, it can be seen that the theory of the five state personality of Yin and Yang in Chinese medicine theory coincides with the frontier theory of modern psychological behavior treatment. It accords with the development of modern medical mode and has wide application prospects.

At the same time, this study also found that there are significant differences in five personality characteristics among different gender of the depressed patients. The scores of the male depressed patients in the dimensions of Tai-yang, Shao-yang, Yin-Yang Balance and Shao-yin are significantly lower than those of female patients, but the scores in the dimension of Tai-yin is significantly higher than those of female patients. Because depression belongs to Yin disease, this fully shows that male depressed patients are more serious than the female patients in pathological abnormalities. But in clinical practice, female patients often have more than male 
patients, which is 2 to 3 times that of male patients. This contradictory phenomena may be caused by the traditional Chinese social culture for men and women's expectation of different roles (men were asked to be more quiet, introverted, forgiving, and so on), which makes the depression of the male depressed patients more occult. According to the relevant investigation shows, about $70 \%$ of male depressed patients do not want to face the fact of depression, which also brings difficulties to early diagnosis and treatment [5].

Of course, because the sample size of this study is too small, it may lead to some limitations in the research results.

\section{SUMMARY}

The personality problem of depressed patients is a very important study. Most researchers believe that the depressed patients have the characteristics of emotional instability in typical introverted personality, and most patients have obvious hypochondria and hysterical personality defects, which is easy to express emotions and stress in the form of somatization. But these studies, as mentioned earlier, are mostly based on western personality measurement tools. The maturity and perfection of personality are influenced by the cultural environment in which individuals grow up. Similarly, the formation and treatment of personality problems can not completely abandon the cultural factors of patients. On the basis of Chinese culture, the personality characteristics of the depressed patients are measured, and their characteristics in the five state personality are found. This provides a matching basis for the treatment of depression by using the theory and methods of Chinese medicine psychology.

The Five State Personality Test Scale is based on the original description of the five state personality in Neijing, combined with the Chinese cultural characteristics and the Chinese norm. Compared with the revised psychological personality scale in the west, its theory, concept system and research method are based on the Chinese local psychology background. Its measurement results are closer to the personality characteristics of Chinese people, and can really describe and explain the behavior and psychological characteristics of Chinese people. Therefore, it helps to further promote the localization process of Chinese psychology by using The Five State Personality Test Scale to investigate and analyze the five state personality of the depressed patients, and using the scale to study and practice.

\section{REFERENCE}

[1] Zhao-yang CHEN. Personality Factors and Depression [J]. Chinese Journal of Behavioral Medicine, 2011,10 (4): 390-391.(In Chinese)

[2] Yong-xin HUANG. Personality Characteristics of Depressed Patients [J]. Cathaysian medicine, 2008, 17 (4): 489-490. (In Chinese)

[3] Kai-mei ZHENG. Study on Five State Personality of Depression [J]. Journal of Tianjin University of Traditional Chinese Medicine, 2013,26 (2): 61-62.(In Chinese)

[4] Meng-han LUI. The Correlation of Personality Characteristics of Depressed Between Five State Personality and Minnesota [D]. Master of Chinese Academy of Chinese medicine, 2010. (In Chinese)

[5] Jing TENG. Research on Personality Characteristics of the Depressed Patients [J]. Chinese Rehabilitation Theory and practice, 2012, 13 (1) 81-82. (In Chinese) 